\title{
Force fluctuation in a driven elastic chain
}

\author{
Frédéric Lacombe, ${ }^{1}$ Stefano Zapperi, ${ }^{1,2}$ and Hans J. Herrmann ${ }^{1}$ \\ ${ }^{1}$ PMMH-ESPCI, 10 rue Vauquelin, 75231 Paris Cedex 05, France \\ ${ }^{2}$ INFM, Dipartimento di Fisica, E. Fermi, Università 'La Sapienza," P.le A. Moro 2, 00185 Roma, Italy
}

(Received 20 July 2000; revised manuscript received 16 November 2000; published 14 February 2001)

\begin{abstract}
We study the dynamics of an elastic chain driven on a disordered substrate and analyze numerically the statistics of force fluctuations at the depinning transition. The probability distribution function of the amplitude of the slip events for small velocities is a power law with an exponent $\tau$ depending on the driving velocity. This result is in qualitative agreement with experimental measurements performed on sliding elastic surfaces with macroscopic asperities. We explore the properties of the depinning transition as a function of the driving mode (i.e., constant force or constant velocity) and compute the force-velocity diagram using finite-size scaling methods. The scaling exponents are in excellent agreement with the values expected in interface models and, contrary to previous studies, we found no difference in the exponents for periodic and disordered chains.
\end{abstract}

DOI: 10.1103/PhysRevB.63.104104

PACS number(s): 64.60.-i, 05.70.Ln, 81.40.Pq

\section{INTRODUCTION}

The dissipative motion of an elastic line in a random potential is an interesting example of a nonequilibrium interacting system and is relevant for several phenomena in condensed matter physics. Examples are numerous and include the motion of magnetic interfaces in ferromagnetic materials, ${ }^{1,2}$ solid friction, ${ }^{3-5}$ wetting, ${ }^{6,7}$ charge-density waves, ${ }^{8}$ fluids in porous media, ${ }^{9}$ vortex dynamics in hightemperature superconductors, ${ }^{10-12}$ cracks, ${ }^{13}$ and dislocations. ${ }^{14}$ These systems are characterized by a dynamic phase transition ruled by the interplay between quenched disorder and elastic interactions. ${ }^{15}$

Due to the effect of the disorder, an elastic chain at zero temperature is pinned when the applied force is below a critical value $F_{c}$ : after a sufficiently long time, independently of the initial conditions, the chain reaches a configuration where no movement is possible. For $F>F_{c}$, the chain can escape from any pinning configuration and moves with constant average velocity. When $F$ is close to $F_{c}$, the motion is dominated by collective effects and the depinning of a single bead produces a large reorganization of the chain. In other words, for $F=F_{c}$, the system is critical and the motion of the beads is highly correlated.

An elastic chain moving in a disordered potential is a useful model to understand some general features of sliding friction, ${ }^{5}$ in particular, of the experiment reported in Refs. 16-18, done using two artificial surfaces with controlled roughness and elasticity. Beads of diameter $2 \mathrm{~mm}$ were randomly put inside an elastic matrix, with a maximum roughness of $0.5 \mathrm{~mm}$. The two surfaces were then displaced against each other at constant velocity and the friction force was measured, varying the elasticity of the matrix and the driving velocity. The distribution of the amplitude of the slip events is generally found to decay as a power law at small velocities, suggesting the presence of an underlying critical point. The exponents characterizing the power-law distribution are found to decrease with the applied velocity, in analogy with other driven systems such as domain walls in ferromagnet. ${ }^{1}$

Several variant of the chain model can be studied in order to reproduce the experiments: periodic or disordered arrangements of beads, ${ }^{4}$ on a rigid ${ }^{4,5}$ or elastic ${ }^{19}$ substrate and the chain can be pulled in its own direction ${ }^{4,5}$ or perpendicular to it. ${ }^{20}$ Simulations of a one-dimensional, periodic or disordered, chain pulled in its own direction over a rigid disordered potential have been performed by Cule and Hwa. ${ }^{4}$ The measurement of the velocity and the roughness exponents indicate that periodic and disordered chains are described by two different universality classes. These and other simulations are performed considering a constant applied force, while experiments are performed driving the system at constant velocity.

Here, considering explicitly both constant velocity [see Fig. 1(a)] and constant force driving [see Fig. 1(b)], we study the force-velocity diagram and measure the force fluctuations as a function of the applied velocity. The distribution of the slips events is power-law distributed, and characterized by an exponent $\tau$, which appears to decrease with the applied velocity, in agreement with the experiments. In our simula-

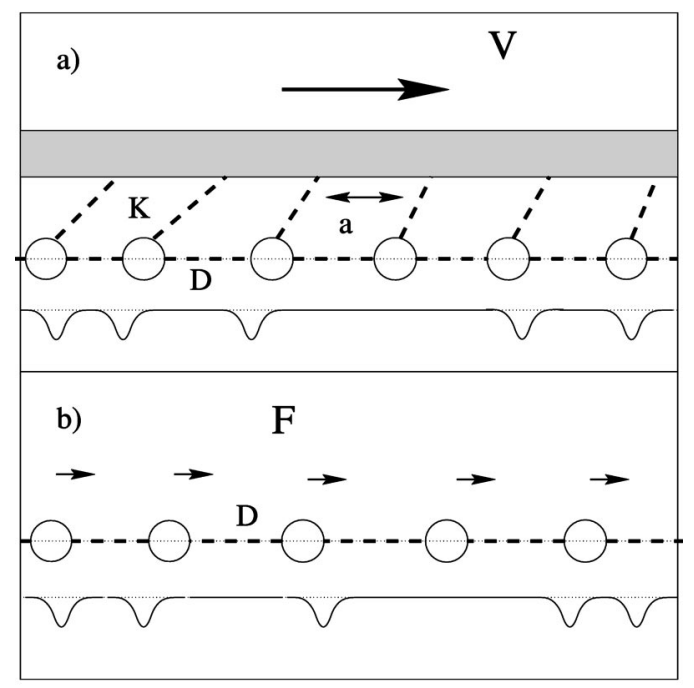

FIG. 1. Schematic motion of the elastic chain driven on a rough substrate at constant velocity (a), attaching the beads to a moving plate via springs of stiffness $K$, and at constant force (b). 
tions, a slip event is identified with the time interval in which the frictional force decreases, and the slip size is defined as in Ref. 16 (see Sec. IV A for more details). In the limit of low velocity, the exponent $\tau$ can be related to the critical exponent obtained tuning the applied force as discussed in Ref. 21 (see also Refs. 1, 4, 22, and 23). We measure the fluctuations in the position of the beads in the constant force and constant velocity cases and obtain the same roughness exponent $\zeta$. Next, we evaluate the force velocity diagram, using finite-size scaling to locate the critical force and compute the exponent $\beta$. The values of the exponents are consistent with scaling relation and in good numerical agreement with the exponents of interface depinning, but disagree with previous simulations for a periodic chain in a random potential. ${ }^{4}$ In order to confirm this conclusion, we simulate the motion of a disordered chain and see no evidence for the existence of two different universality classes for periodic and disordered chains, in disagreement with the conclusions of Ref. 4.

The paper is organized as follows; in Sec. II we introduce the model, and in Sec. III we define the critical exponents and discuss some scaling relations. In Sec. IV A we present the numerical results obtained at constant velocity, in Sec. IV B we discuss the constant force case studying the scaling behavior close to the depinning transition. In Sec. V we summarize the main results of the paper.

\section{MODEL}

We consider the overdamped dynamics of a onedimensional line of elastically coupled beads, driven on a disordered substrate. The disordered potential is a succession of identical Gaussian potentials, randomly distributed in space. The beads can be driven directly, applying a constant force, or indirectly coupling them to an intermediate spring which is pulled at constant velocity. The equation of motion is,

$$
\eta \frac{\partial r_{i}(t)}{\partial t}=D\left(r_{i+1}-2 r_{i}+r_{i-1}\right)+f\left[r_{i}(t)\right]+F,
$$

where $r_{i}(t)$ is the position of the bead $i$ at time $t, \eta$ is the coefficient of viscosity, $D$ is the stiffness of the elastic line, $F$ is the driving force, and $f(x)$ is a random force, due to the contribution of the ensemble of pinning centers. We model the random force by the sum of $N$ derivatives of Gaussian potentials located on the pinning site

$$
f(x)=C \sum_{i=1}^{N}\left(x-x_{i}^{p}\right) \exp \left[-\frac{1}{2} \frac{\left(x-x_{i}^{p}\right)^{2}}{\sigma^{2}}\right],
$$

where $C$ represents the strength of the disorder, $\sigma$ quantifies the width of the wells, and $x_{i}^{p}$ is the location of the pinning site $i$, which we chose to be Poisson distributed. The equation of motion is integrated numerically using a fourth-order Runge-Kutta method.

The interplay between disorder and elastic interactions in our model can be understood computing the Larkin length $l_{L} \cdot{ }^{24}$ For distances smaller than $l_{L}$, the beads are interacting strongly and the chain moves coherently, while for distances larger than $l_{L}$, the random forces become dominant and the chain deforms considerably. The Larkin length can be estimated considering both the effect of the rigidity of the line and the strength of the disorder, and for our model it is given by $^{5}$

$$
l_{L} \approx\left[\frac{D a \sigma^{2}}{\rho^{1 / 2} C}\right]^{2 / 3},
$$

where $a$ is the distance between the beads and $\rho$ is the density of pinning centers. In order to analyze the critical properties of the system, we have to consider the limit where the Larkin length is larger than the mean distance between the beads and the dynamics is governed by the collective motion of the beads. To this end, we carefully choose the parameters of the model so that $l_{L} \gg a$.

\section{SCALING RELATIONS}

Depending on the method used to drive the chain, the measured quantities change, but the corresponding critical exponents can be related by scaling relations. Here we summarize the scaling properties of the depinning transition in the case where the beads are driven by springs of stiffness $K$ pulled at constant velocity $V$ and in the case where they are submitted to a constant force $F$.

\section{A. Constant velocity driving}

Friction experiments are generally performed under a constant velocity driving. In some cases, a traction machine turning at constant velocity is coupled by a spring to the sliding system. In other cases, an effective spring coupling is due to the elastic deformation of the material driven imposing a constant strain rate far from the sliding interface. To simulate constant velocity driving, ${ }^{25}$ we attach each bead to a spring of stiffness $K^{4}$, so that the force is given by

$$
F=K\left[r_{i}(0)+V t-r_{i}(t)\right],
$$

where $V$ is the applied velocity [see Fig. 1(a)]. When the velocity $V$ and the stiffness $K$ are small, the motion displays large fluctuations. In particular, in the limit $V \rightarrow 0$ and $K$ $\rightarrow 0$ the system reaches the depinning transition and the force fluctuates around $F_{c}$. This feature is common to other nonequilibrium critical phenomena, such as absorbing state phase transitions and self-organized criticality. ${ }^{26,27}$

The distribution of the friction force fluctuations is directly related to the size distribution of slip events $\Delta x$ of the chain, since $\Delta F=K \Delta x$. Here $x \equiv \Sigma_{i} r_{i}$ and the slip is defined by a drop in the measured friction force (see Fig. 1). Close to the depinning transition, we expect that the distribution of $\Delta x$ decays as

$$
P(\Delta x) \sim \Delta x^{-\tau} h\left(\Delta x / \Delta x_{0}\right),
$$

where $h(x)$ is a scaling function and $\Delta x_{0}$ the cutoff value. The value of the cutoff depends on various parameters, such as the system size employed in the simulation. Clearly the avalanche size cannot be greater than the total length of the 

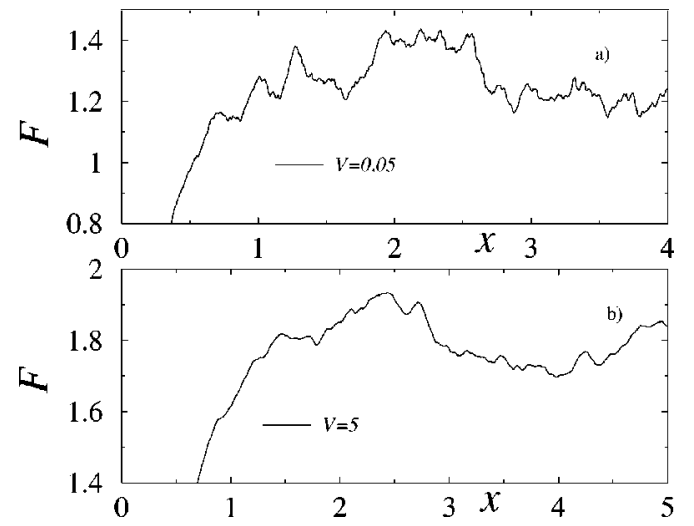

FIG. 2. Friction force as a function of the displacement for different loading speeds. (a) $V=0.05$, (b) $V=5$.

line. Furthermore, we expect that the stiffness of the springs $K$ and the driving velocity $V$ will, in general, change the value of the cutoff. In the low velocity limit and for large enough system sizes, $K$ becomes the dominant parameter that determines the value of the cutoff. ${ }^{2,4}$

The scaling of the cutoff with $K$ can be used to evaluate the roughness exponent $\zeta$, using the relation

$$
\Delta x_{0} \sim K^{-\zeta / 2},
$$

which results from the fact that the correlation length scales with $K$ as $\xi_{0} \sim K^{-1 / 2}$ (Ref. 4) and by definition $\Delta x_{0} \sim \xi_{0}^{\zeta}$. This result implies that $P(\Delta x, K)$ should satisfy the scaling form

$$
P(\Delta x, K) K^{-\tau \zeta / 2}=H(t), \quad t \equiv \Delta x K^{\zeta / 2} .
$$

Equation (5) is used to compute $\tau$ and Eqs. (6) and (7) are used to compute the exponent $\zeta$.

The exponent $\zeta$ can also be evaluated directly using the scaling of the fluctuations of the displacements with $K$. Defining the relative displacements of the beads as $u_{i}(t)$ $\equiv r_{i}(t)-\left[V t+r_{i}(0)\right]$, the fluctuations can be quantified by

$$
W^{2}=\sum_{i=1}^{L}\left[u_{i}(t)-m(t)\right]^{2} / L,
$$

where $m(t) \equiv \sum_{i=1}^{L} u_{i}(t) / L$, and $L$ is the number of beads. The roughness $W$ scales with the correlation length $\xi_{0}$ as $W \sim \xi_{0}^{\zeta}$, and since $\xi_{0} \sim K^{-1 / 2}$

$$
W \sim K^{-\zeta / 2} .
$$

Equations (6) and (9) have the same origin and can independently be used to estimate $\zeta$.

\section{B. Constant force driving}

When the system is driven at constant force [see Fig. 1(b)], we expect a depinning transition as a function of $F$. For $F>F_{c}$, the chain moves with constant average velocity $v$ defining an exponent $\beta$

$$
v \sim\left(F-F_{c}\right)^{\beta} .
$$

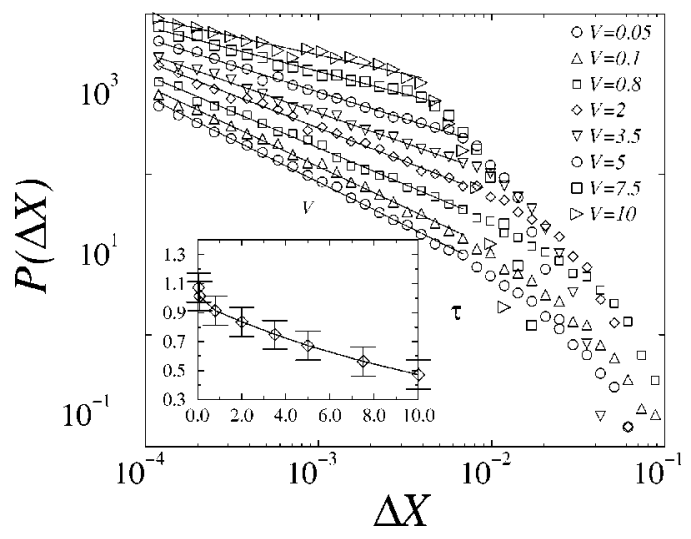

FIG. 3. Log-log plot of the size distribution of slip events for different values of $V$. In the inset we report the exponent $\tau$, obtained fitting the distributions, as a function of the driving velocity.

Close to the depinning transition the motion is very irregular, and large regions of the chain move collectively. The correlation length diverges at the transition as

$$
\xi \sim\left(F-F_{c}\right)^{-\nu} .
$$

In order to estimate the critical exponents $\beta$ and $\nu$, we employ a particular finite-size scaling method, ${ }^{13}$ in analogy with absorbing state phase transitions. ${ }^{28}$

We first compute the critical force analyzing the decay of the average velocity with time for different system sizes. For finite systems, the average velocity reaches a quasi steady state $v(F, L)$. When $F>F_{c}$ we expect that as the size $L$ $\rightarrow \infty, v(F, L)$ approaches a nonvanishing value given by Eq. (10), while it decays to zero for $F<F_{c}$. At the depinning transition we expect that

$$
v\left(F_{c}, L\right) \sim L^{-\beta / \nu} .
$$

Once $F_{c}$ is known with good precision, we can measure directly the exponent $\beta$ from Eq. (10). As in the constant velocity case, we can evaluate the roughness exponent, measuring the width at $F_{c}$ as a function of $L$, which should scale as

$$
W(L) \sim L^{\zeta}
$$

For a periodic chain, Ref. 4 reported $\beta \simeq 0.4$ and $\zeta \simeq 1.5$, while for a disordered $\beta \simeq 0.25$ and $\zeta \simeq 1.2$. These last values are consistent with interface depinning that in $d=1$ yields $\beta \simeq 0.25$ and $\zeta \simeq 1.25$. $^{29}$

\section{NUMERICAL RESULTS}

\section{A. Constant velocity}

The primary interest of this study is to compute the exponent $\tau$ which characterizes the collective motion of the particles at $F \simeq F_{c}$, and in particular its dependence on the driving velocity. We note that the avalanche exponent was found to decrease with the driving velocity in the Barkhausen effect, due to the motion of domain walls in a ferromagnet. ${ }^{1}$ The same effect was observed in the friction experiments reported in Ref. 16. 
TABLE I. Critical exponents measured in simulations.

\begin{tabular}{cc}
\hline \hline$\tau$ & $1.07 \pm 0.05$ \\
$\zeta$ & $1.26 \pm 0.03$ \\
$\beta$ & $0.22 \pm 0.02$ \\
$\nu$ & $1.3 \pm 0.1$ \\
\hline
\end{tabular}

In order to reach the scaling regime, we progressively decrease $V$ and $K$ and compute the friction force. Figure 2 shows two typical plot for the friction force as a function of the position of the line, in Fig. 2(a) the driving velocity is $V=0.05$ and in Fig. 2(b) $V=5$. As the driving velocity increases, the friction force becomes smoother, and in the limit $V \gg 1$ we obtain a viscous behavior $(F \sim \eta V)$ with small relative fluctuations. On the contrary, for small velocities the dynamics is jerky: the force increases with time until the beads are sufficiently stressed so that the chain depins decreasing the force.

We measure the friction force drops $\Delta F$, or the slip sizes $\Delta X=\Delta F / K$ and analyze their distribution. These quantities are numerically calculated as follows: when the friction force starts to decrease, we record its value $F_{\max }$ and wait until the friction force increases again, defining a value $F_{\min }$. The friction force drop is simply given by $\Delta F=F_{\max }-F_{\text {min }}$. The distributions are averaged over 20 realizations of the disorder for $V=0.05$ and 100 realizations for $V=10$; in all cases the system was composed of $L=1000$ beads, and the disorder was produced by $N=20000$ pinning sites, Poissonian distributed. The value of the exponent $\tau$ is obtained by a direct fit of the linear part of the distribution plotted in a $\log$-log graph. The results are shown in Fig. 3, the main graph presents the log-log plot of the probability distribution function of the jumps for various $V$ and in the inset we report the value of $\tau$ as function of the velocity. We see a slow decrease of the exponent when the velocity increases. This result is in good qualitative agreement with the experiments reported in Ref. 16. The value of $\tau$ for $V \rightarrow 0$ (see Table I) agrees well with the exponent obtained for elastic line depinning under quasistatic conditions, ${ }^{23,30}$ considering, however, the motion perpendicular to the line direction.

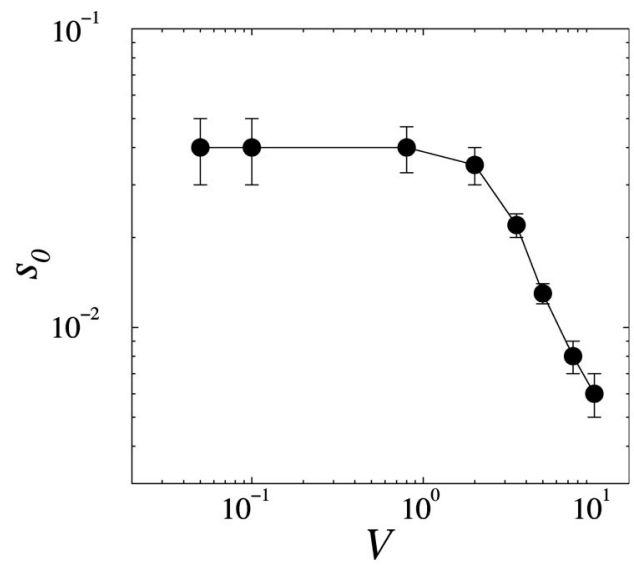

FIG. 4. Cutoff of the slip size distribution as a function of $V$. For sufficiently low velocities $\left(V<V^{*}\right)$, the cutoff is independent of $V$.

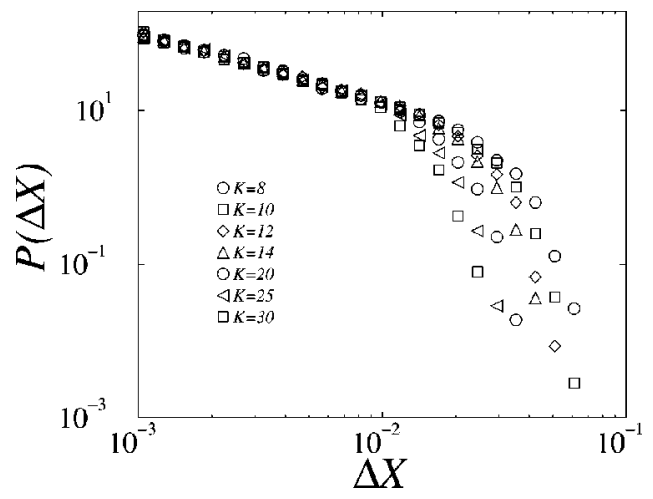

FIG. 5. Distribution of the slips events as a function of $K$ showing that the cutoff increases when $K$ decreases. The velocity $V$ is kept constant.

Figure 3 shows that the cutoff $\Delta X_{0}$ clearly depends on $V$ when $K$ is hold fixed. We can quantify this variation and the result is reported in Fig. 4. For small velocities $\left(V<V^{*}\right)$ the cutoff is a constant (i.e., it does not depend on $V$ ) which in principle depends on $K$, while for high velocities the cutoff decreases with $V$, roughly as a power law. Next, we study the behavior of the cutoff when $V<V^{*}$ as $K$ is varied. In Fig. 5 we show the distribution of slip sizes for various $K$ for very small driving velocity. We see that the cut off increases as $K$ is decreased. Figure 6 shows the collapse of the curves after the rescaling with $K$, in accordance with Eq. (7).

We also measure the roughness exponent of the system following Eq. (9) and the result is reported in Fig. 7. We obtain with both methods $\zeta=1.26$, which is consistent with the numerical value found in interface depinning, ${ }^{29}$ for a disordered chain ${ }^{4}$ and with a recent two loop renormalization group calculation. ${ }^{31}$ This value, nevertheless, disagrees with previous results on a periodic chain. ${ }^{4}$

The simulations of Ref. 4 for periodic and disordered chain, suggest the presence of two different universality classes. In order to test this result we study the force fluctuations of a disordered chain. The equilibrium length of the springs connecting the beads is chosen randomly (Poisson). The chain is then driven at constant velocity and the distribution of the slip sizes is calculated. The result is shown in Fig. 8 where we also report the distribution obtained with a periodic chain using similar parameters. The two distribu-

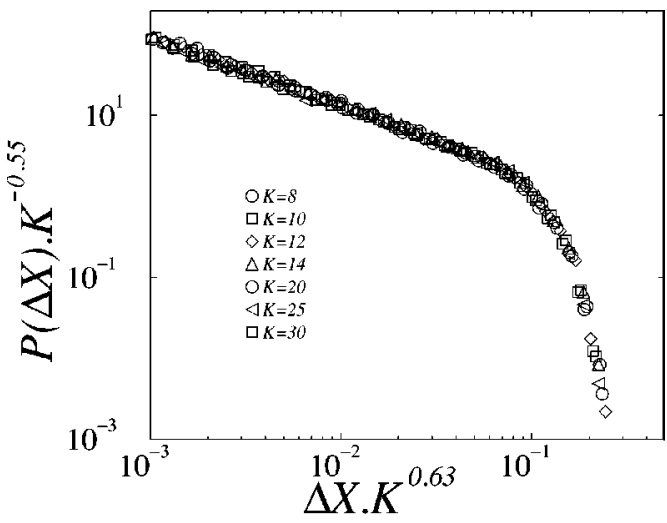

FIG. 6. Data collapse of Fig. 5. 


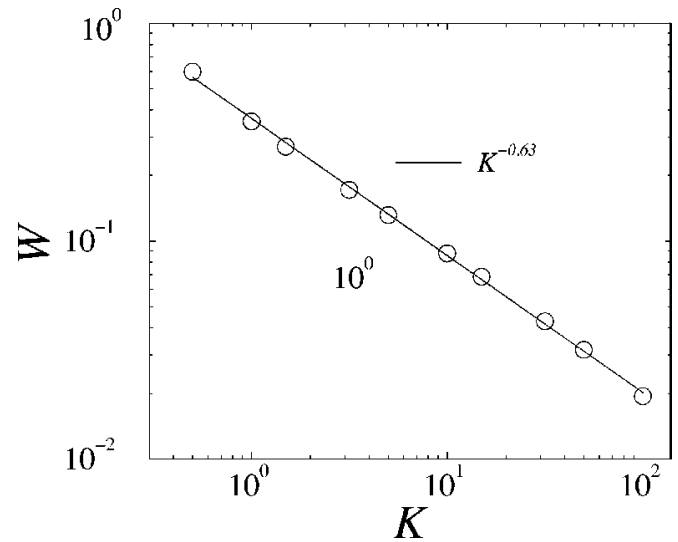

FIG. 7. Width of the beads displacements as a function of $K$. The power-law fit of the curve and the scaling relations imply that $\zeta=1.26 \pm 0.03$.

tions are clearly indistinguishable, casting some doubt on the relevance of disorder in the spring lengths.

The only difference between the simulations presented in Ref. 4 and ours lies in the way disorder is implemented: in Ref. 4 the pinning points are arranged in a periodic structure and have random strength, while we use constant strength and random positions. In the case of a periodic chain, Cule and $\mathrm{Hwa}^{4}$ measure $\zeta \simeq 1.5$, which is expected below the Larkin length, although the parameter employed do not seem to be consistent with that regime. To check whether the difference in the pinning field is relevant or not for the critical behavior, we measure the fluctuations of the chain position as a function of $K$ for a periodic chain sliding on a substrate in which pinning centers are arranged periodically and have random strength. The result reported in Fig. 9 clearly shows that the roughness exponent $\zeta=1.26 \pm 0.05$ does not change with respect to case studied earlier. From this study, we conclude that the two models are in the same universality class.

\section{B. Constant force}

For constant force driving, we employ system sizes varying from $L=20$ to $L=540$ and the density of pinning sites

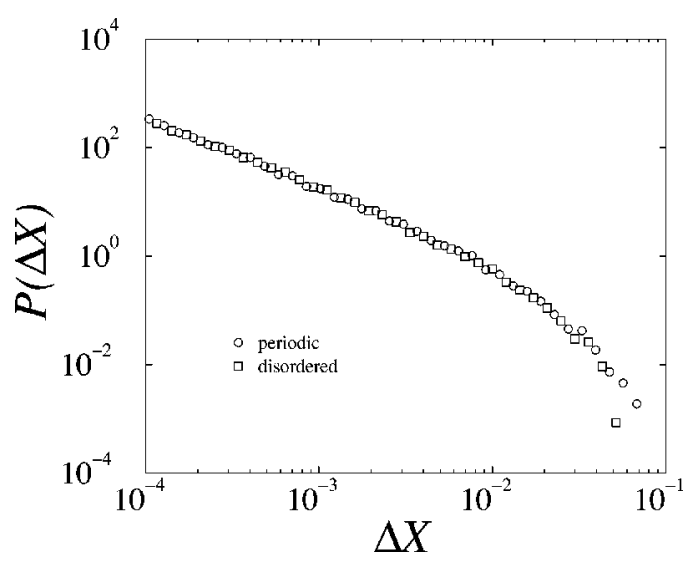

FIG. 8. Probability distribution of slip sizes for a periodic and a disordered chain. We see no evidence for the existence of two different classes, since the distributions are indistinguishable.

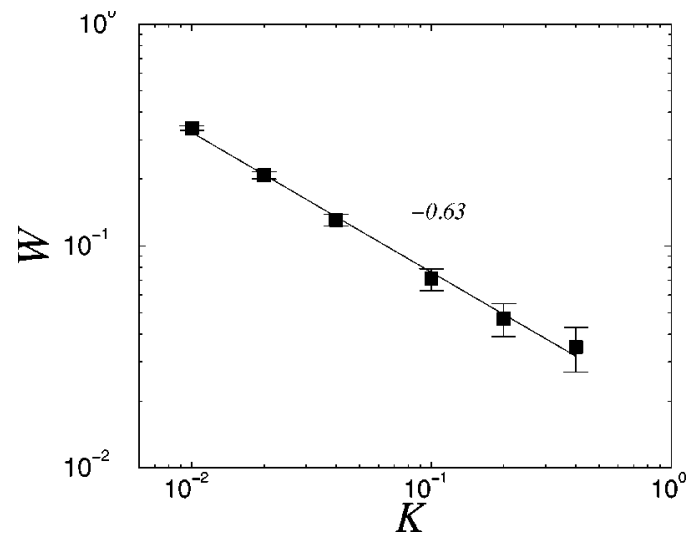

FIG. 9. Width of the beads displacements as a function of $K$ for a periodic chain sliding on a substrate where pinning centers are arranged periodically and have random strength (Ref. 4). The power-law fit of the curve and the scaling relations imply that $\zeta$ $=1.26 \pm 0.05$.

was chosen equal to unity $(L=N)$. In order to determine the exponent $\beta$, we need an accurate estimate of the critical force, since an error in $F_{c}$ can strongly bias the fit.

Figure 10 shows the value of the average velocity of the interface as a function of $L$. For $F$ smaller than $F_{c}$ in the limit of a large system $v$ tends to zero, and for $F$ greater than $F_{c}$ and for the same limit $(L \gg 1) v$ should tend a nonvanishing value. In this way we can locate the critical force, which results to be $F_{c}=2.195 \pm 0.005$. This result appears clearly from Fig. 10, from the $\log -\log$ plot of $v$ as a function of $L$. We see that $F=2.195$ is compatible with a power-law behavior, whereas for $F=2.200$ in the large $L$ limit the mean velocity tends to a nonzero constant. For $F=2.190$ the velocity tends to zero faster than a power law in the limit of large system sizes. The numerical results are averaged over a number of disorder configurations which varies from 4000 for $L=20$ to 200 for $L=200$. The curve plotted in Fig. 10 allows also to estimate $\beta / \nu=0.16 \pm 0.02$ [see Eq. (12)].

Next, we calculate the exponent $\beta$ directly, plotting $v$ vs $\left(F-F_{c}\right)$. The fit in Fig. 11 yields $\beta=0.22 \pm 0.02$. We re-

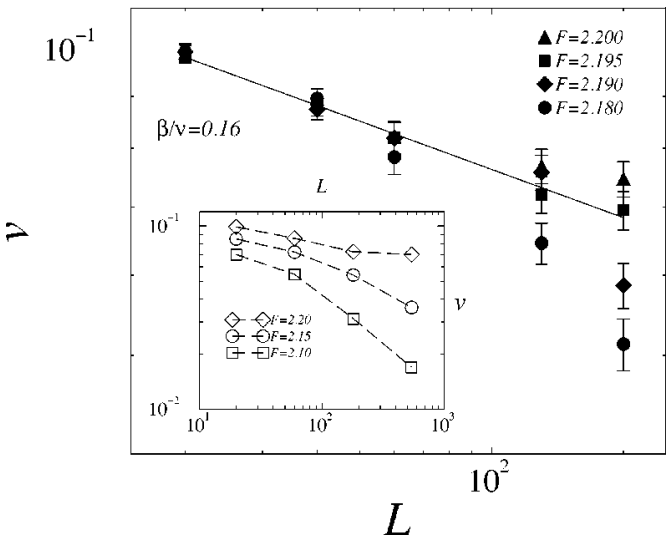

FIG. 10. The variation of the mean velocity $v$ of the chain as a function of the number of beads $L$ and for various forces $F$. The inset shows the data for $F$ far from the critical force $F_{c}$, and the main plot shows the data for $F$ near the critical force, $F_{c} \approx 2.195$. 


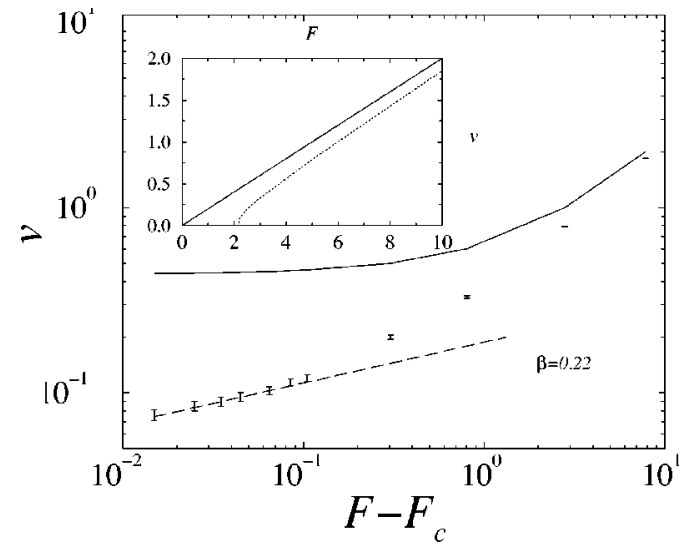

FIG. 11. Average velocity $v$ as a function of the force $F$. The inset shows the $v-F$ diagram. The solid curve represents the result without pinning centers and the dotted line is the result for the disordered substrate. The main graph represents the log-log plot of $v$ as a function of $F-F_{c}$. The exponent $\beta$ is calculated using the six first points of the curve and the exponent is $\beta=0.22 \pm 0.02$.

strict the fit to the six smaller values since a crossover to linear behavior is expected at high forces (i.e., $v=F / \eta$ ) and this can bias the numerical estimate of the exponent. The simulations are made with a system of $L=540$ particles, and the results are averaged over 100 configurations of the disorder. In this way, we can obtain $\beta$ and $\nu$ as summarized in Table I.

To further test the consistency of our results, we calculate the exponent $\zeta$ measuring the scaling of $W$ with $L$ at $F$ $=F_{c}$ [see Eq. (13)]. The results shown in Fig. 12 give $\zeta$ $=1.28 \pm 0.03$ in agreement with the result obtained in Sec. IV A.

\section{CONCLUSION}

In conclusion, we have investigated the dynamics of an elastic chain sliding on a disordered substrate. We have analyzed numerically the scaling close to the depinning transition focusing on the effect of different driving modes. Usually, models are analyzed under constant force driving, while friction experiments are often performed controlling the velocity. The two cases are closely related as discussed in Refs. $2,4,21,23$, and 27 . We have computed the critical exponents characterizing the transition and analyze the effect of the driving velocity and the loading spring stiffness. Our results are in qualitative agreement with friction experiments performed with macroscopic asperities coupled by an elastic matrix. ${ }^{16}$ The experiments reported in Ref. 16 showed a variation of the value of $\tau$ with the type of material used for the matrix. The values computed in our simulations seem to

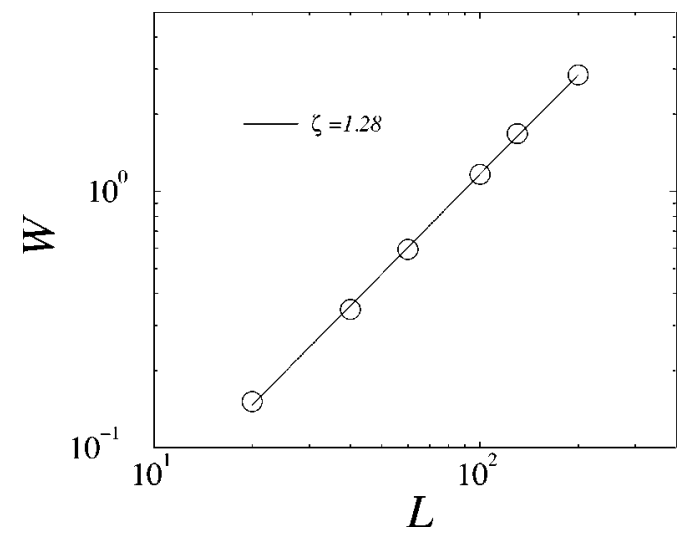

FIG. 12. Width of the beads displacements $W$ as a function of $L$. The scaling relation between the two quantities gives $\zeta$. We recover a value in good agreement with our previous results.

agree quantitatively for a wide range of velocities with the case of a soft-rigid interface, which should correspond to an elastic chain sliding on a rigid rough substrate. In our model, we do not consider inertial effects and vertical displacements which in principle are present in experiments. In addition, it is important to remark that our model will not apply to generic friction experiments ${ }^{3}$ since in many instances the Larkin length is extremely large. ${ }^{32}$

The exponents we measure agree well with the values expected for the depinning of elastic interfaces in quenched disordered media ${ }^{29}$ and with there normalization group calculation of Ref. 31, despite the fact that in our case the line is driven parallel to its direction, while in Refs. 29, 30, and 31, the motion is perpendicular to the line direction. Parallel motion generates memory effects that could change the universality class, but our simulations show that this is not the case. Using the method discussed in Ref. 12, we can show that the continuum limit of the model we study is described by

$$
\frac{\partial h(x, t)}{\partial t}+v \frac{\partial h}{\partial x}=D \nabla h+F+f_{p}(x, h),
$$

where $h(x, t)$ is a coarse grained version of $u_{i}(t), v$ is the average velocity of the beads and $f_{p}$ is a coarse grained random pinning force. Notice that Eq. (14) contains a convective term that is not present in interface depinning models. $^{22,23,29,31}$ The present simulations indicate that this term is not relevant for the critical behavior.

\section{ACKNOWLEDGMENTS}

We thank J. Vannimenus, P. Chauve, and K. Wiese for useful remarks. S.Z. acknowledges financial support from EC TMR Research Network under Contract No. ERBFMRXCT960062.

\footnotetext{
${ }^{1}$ S. Zapperi, P. Cizeau, G. Durin, and H. E. Stanley, Phys. Rev. B 58, 6353 (1998).

${ }^{2}$ G. Durin and S. Zapperi, Phys. Rev. Lett. 84, 4705 (2000).

${ }^{3}$ B.N.J. Persson, Sliding Friction (Springer, Berlin, 1998).
}

${ }^{4}$ D. Cule and T. Hwa, Phys. Rev. Lett. 77, 278 (1996); Phys. Rev. B 57, 8235 (1998).

${ }^{5}$ L. Bocquet and H. J. Jensen, J. Phys. I 7, 1603 (1997); H. J. Jensen, Y. Brechet, and B. Doucot, ibid. 3, 611 (1993). 
${ }^{6}$ D. Ertas and M. Kardar, Phys. Rev. E 49, R2532 (1994).

${ }^{7}$ E. Raphaël and P. G. de Gennes, J. Chem. Phys. 90, 7577 (1989).

${ }^{8}$ G. Parisi and L. Pietronero, Europhys. Lett. 16, 321 (1991); Physica A 179, 16 (1991); O. Narayan and D. Fisher, Phys. Rev. B 46, 11520 (1992).

${ }^{9}$ H. Ji and M. O. Robbins, Phys. Rev. A 44, 2538 (1991).

${ }^{10}$ D. Ertas and M. Kardar, Phys. Rev. B 53, 3520 (1996).

${ }^{11}$ S. Zapperi, J. S. Andrade, Jr., and J. Mendes Filho, Phys. Rev. B 61, 14791 (2000).

${ }^{12}$ T. Giamarchi and P. Le Doussal, Phys. Rev. B 52, 1242 (1995).

${ }^{13}$ J. Schmittbuhl, S. Roux, J. P. Vilotte, and K. J. Måløy, Phys. Rev. Lett. 74, 1787 (1995).

${ }^{14}$ S. Zapperi and M. Zaiser, cond-mat/0011083 (unpublished).

${ }^{15}$ For a review, see M. Kardar, Phys. Rep. 301, 85 (1998).

${ }^{16}$ S. Ciliberto and C. Laroche, J. Phys. I 4, 223 (1993).

${ }^{17}$ S. Ciliberto and C. Laroche, Eur. Phys. J. B 9, 551 (1999).

${ }^{18}$ J. Crassous, S. Ciliberto, E. Charlaix, and C. Laroche, J. Phys. II 7, 1745 (1997).

${ }^{19}$ T. Kawaguchi and H. Matsukawa, Phys. Rev. B 56, 13932 (1997); 58, 15866 (1998).

${ }^{20}$ A. Tanguy, M. Gounelle, and S. Roux, Phys. Rev. E 58, 1577 (1998).
${ }^{21}$ C. Tang and P. Bak, Phys. Rev. Lett. 60, 2347 (1988).

${ }^{22}$ O. Narayan and D. Fisher, Phys. Rev. B 48, 7030 (1993).

${ }^{23}$ M. Paczuski, S. Maslov, and P. Bak, Phys. Rev. E 53, 414 (1996).

${ }^{24}$ A. I. Larkin and Y. N. Ovchinnikov, J. Low Temp. Phys. 74, 409 (1979).

${ }^{25}$ Another possibility is to drive the chain quasistatically with an extremal dynamics (Ref. 23). The velocity is then constant and equal to $1 / L$ and the chain approaches the critical point.

${ }^{26}$ P. Bak, C. Tang, and K. Wiesenfeld, Phys. Rev. Lett. 59, 381 (1987).

${ }^{27}$ For a review, see R. Dickman, M. A. Muñoz, A. Vespignani, and S. Zapperi, Braz. J. Phys. 30, 27 (2000), and references therein.

${ }^{28}$ I. Jensen and R. Dickman, Phys. Rev. E 48, 1710 (1993).

${ }^{29}$ H. Leschhorn, T. Nattermann, S. Stepanow, and L.-H. Tang, Ann. Phys. 6, 1 (1997).

${ }^{30}$ S. Roux and A. Hansen, J. Phys. I 4, 515 (1994).

${ }^{31}$ P. Chauve, P. Le Doussal, and K. Wiese, cond-mat/0006056 (unpublished).

${ }^{32} \mathrm{C}$. Caroli and $\mathrm{Ph}$. Nozières, in The Physics of Sliding Friction, Vol. 311 of NATO Advanced Study Institute Series B: Physics, edited by B.N.J. Persson (Kluwer, Dordrecht, 1996); Eur. Phys. J. B 4, 23 (1998). 